\title{
OPEN Methods for tuning plasmonic and photonic optical resonances in high surface area porous electrodes
}

\author{
Lauren M. Otto ${ }^{1,2,3}$, E. Ashley Gaulding ${ }^{3,4}$, Christopher T. Chen², Tevye R. Kuykendall', \\ Aeron T. Hammack ${ }^{2}$, Francesca M. Toma ${ }^{3,4}$, D. Frank Ogletree ${ }^{2}$, Shaul Aloni ${ }^{2}$, \\ Bethanie J. H. Stadler ${ }^{1}$ \& Adam M. Schwartzberg ${ }^{2 \bowtie}$
}

Surface plasmons have found a wide range of applications in plasmonic and nanophotonic devices. The combination of plasmonics with three-dimensional photonic crystals has enormous potential for the efficient localization of light in high surface area photoelectrodes. However, the metals traditionally used for plasmonics are difficult to form into three-dimensional periodic structures and have limited optical penetration depth at operational frequencies, which limits their use in nanofabricated photonic crystal devices. The recent decade has seen an expansion of the plasmonic material portfolio into conducting ceramics, driven by their potential for improved stability, and their conformal growth via atomic layer deposition has been established. In this work, we have created three-dimensional photonic crystals with an ultrathin plasmonic titanium nitride coating that preserves photonic activity. Plasmonic titanium nitride enhances optical fields within the photonic electrode while maintaining sufficient light penetration. Additionally, we show that post-growth annealing can tune the plasmonic resonance of titanium nitride to overlap with the photonic resonance, potentially enabling coupled-phenomena applications for these three-dimensional nanophotonic systems. Through characterization of the tuning knobs of bead size, deposition temperature and cycle count, and annealing conditions, we can create an electrically- and plasmonically-active photonic crystal as-desired for a particular application of choice.

The term "plasmonics" was initially coined as an analogous term to "photonics" and "electronics", after early researchers realized that surface plasmons could enable a new class of devices based on their unique properties ${ }^{1,2}$. Surface plasmons are electron density waves that are bound to the interface of a metallic and a dielectric material and are induced by incoming resonant photons. Surface plasmons generate strong, high-gradient electric fields that are particularly sensitive to the surrounding media and have been applied to circuits and inter-device communications $^{3,4}$, biological- and chemical-sensing and imaging ${ }^{5-7}$, precision light sources ${ }^{8-10}$, photovoltaics $^{11,12}$, $\mathrm{CO}_{2}$ reduction ${ }^{13-16}$, solar water splitting ${ }^{17-20}$, and as nanoscale heaters for heat-assisted magnetic recording data storage $^{21,22}$.

To investigate how plasmons interact with other photonic and electronic phenomena in three-dimensional architectures $^{23,24}$, we began investigating high surface area, plasmonically-active photonic crystal electrodes. Similar to the way periodic atomic arrangement controls electron propagation, periodic nanostructured features can be used to transmit or block certain light frequencies in photonic crystals ${ }^{25,26}$. Photonic crystals have enabled many advanced technologies including microlasers ${ }^{27}$, light emitting diodes ${ }^{28}$, dye-sensitized solar cells ${ }^{29}$, and functional built-in elements and optical fibers for telecommunications $\mathrm{s}^{30,31}$. Templated opal and inverse opal structures are frequently used in photonic crystal applications due to their relative ease of fabrication ${ }^{32-34}$. However, creating high surface area photonic crystals with conducting surfaces is challenging. Many applications can benefit from such structures, including emitters for electrically tunable color films ${ }^{34-36}$ and thermophotovoltaics ${ }^{37-39}$ as well as high surface area electrodes for solar water splitting ${ }^{17,40}$ and supercapacitors ${ }^{41,42}$. Furthermore, combining

${ }^{1}$ Department of Electrical and Computer Engineering, University of Minnesota, Minneapolis, USA. ${ }^{2}$ The Molecular Foundry, Lawrence Berkeley National Laboratory, Berkeley, USA. ${ }^{3}$ Joint Center for Artificial Photosynthesis, Lawrence Berkeley National Laboratory, Berkeley, USA. ${ }^{4}$ Chemical Sciences Division, Lawrence Berkeley National Laboratory, Berkeley, USA. ${ }^{\square}$ email: ams@Ibl.gov 
the properties of both photonic light trapping and plasmonic field enhancement in a single material, it may be possible to increase the efficiency of a variety of the aforementioned optically driven processes including light harvesting materials and devices. A major challenge, however, is the large increase in absorption and reflection that occur as plasmonic effects increase. This phenomenon may dampen or destroy the photonic resonance, negating any cooperative effects. In some cases, conductive and plasmonic materials like silver may enable the desired quality of photonic crystals ${ }^{43}$; however, creating such complex metallic photonic crystals is challenging because noble metals are soft and mechanically unstable, whereas hard metals are reactive, and forming continuous ultrathin $(<7 \mathrm{~nm})$ metal layers into three-dimensional structures is difficult to achieve.

TiN is a highly conductive and plasmonically active ceramic with excellent chemical and mechanical stability $^{44,45}$. For over 20 years, TiN has been used for conformal and three-dimensional ultrathin conducting films using thermal atomic layer deposition (ALD) and plasma-enhanced ALD (PEALD) at the industrial scale $^{46,47}$. Despite a long history of application of ALD and PEALD to creating or coating photonic crystals with a diverse variety of high quality materials over complex geometries, ALD TiN-based three-dimensional conductive photonic crystals have not been explored to the best of our knowledge $e^{48-53}$.

In this work, we demonstrate the ability to produce high surface area, porous, bicontinuous, three-dimensional, photonic crystal structures that are capable of trapping and controlling light using conductive, plasmonic TiN conformally coated onto a $\mathrm{SiO}_{2}$ photonic crystal by PEALD. Conformal coatings of TiN as thin as $2 \mathrm{~nm}$ were used to study the plasmonic, photonic, and electronic properties of an ultrathin film applied to a non-conducting photonic crystal. The TiN-coated structure is both a conducting photonic crystal and a high surface area electrode with possible applications utilizing the plasmonic, photonic, and electronic effects. We use thermal treatments to demonstrate simultaneous tuning of the plasmonic, photonic, and ohmic response.

\section{Results and discussion}

Conventionally, inverse opal structures are formed by fully infilling the void space in self-assembled opal templates, followed by removal of the template. In order to produce extremely high surface area electrodes with both photonic and plasmonic properties, we have developed a partially infilled inverse opal, effectively doubling the surface area over conventional inverse opals, layered with a conductive and plasmonic TiN thin film that coats all surfaces. For simplicity, we refer to this structure as an inverse opal. The fabrication process and resulting structure are shown in Fig. 1 following the initial self-assembly of a polystyrene opal template as previously demonstrated $^{33}$. In principle, one could coat this opal with PEALD grown TiN and obtain a high surface area, plasmonic, photonic crystal electrode; however, the polystyrene template is unstable above $100{ }^{\circ} \mathrm{C}$ due to close proximity to the polymer-glass transition, and TiN grown below this temperature is relatively low quality. In addition, extremely thin TiN films will be necessary in order to obtain the required optical transmission, such that structural stability would likely be compromised. We are able to overcome all of these challenges by first coating the opal template with silica, followed by removal of the polystyrene template by annealing in air, and finally performing PEALD of TiN on the $\mathrm{SiO}_{2}$ template at temperatures near $300^{\circ} \mathrm{C}$. The result is a low density, inverse opal structure with significantly enhanced surface area. Figure $1 \mathrm{~b}$ shows a scanning electron microscopy (SEM) image of a sample with a $47 \mathrm{~nm}$ TiN coating.

To explore the optical and electronic quality of the as-deposited TiN, we used in situ spectroscopic ellipsometry (SE) to monitor the growth process on planar substrates (silicon with $250 \mathrm{~nm}$ of thermally grown $\mathrm{SiO}_{2}$ ). Ex situ variable-angle SE (VASE, supplementary Figure S1) was used for more detailed analysis of air-exposed samples, and van der Pauw measurements were used to directly probe the conductivity of films deposited on the planar substrates. Ex situ VASE data was acquired at 5 degree intervals between 45 and 75 degrees and analyzed using a parametric fit to a Drude-Lorentz model from which we extract the films' dielectric function and conductivity ${ }^{5-56}$. The best fit was achieved by including an approximately $1.5 \mathrm{~nm}$ thick $\mathrm{TiO}_{2}$ layer and correspondingly reduced TiN thickness. This native oxide layer forms upon exposure to air and is not observed in the in situ SE measurements. Figure 2a,b show the real and imaginary parts, respectively, of the dielectric function obtained by ex situ VASE of films ranging from 2 to $47 \mathrm{~nm}$ (all thicknesses measured by ex situ VASE). The 2.0 and $3.5 \mathrm{~nm}$ thick films do not demonstrate plasmonic behavior as $\epsilon_{m}^{\prime}$ is positive across all measured wavelengths. A transition to plasmonic behavior is observed for thicknesses above $3.5 \mathrm{~nm}$. The $7.6 \mathrm{~nm}$ thick TiN film becomes weakly plasmonic ( $\epsilon_{m}^{\prime}$ is negative) for much of the red and near infrared portions of the spectrum. The lack of plasmonic behavior in ultrathin $(<7 \mathrm{~nm})$ films is likely a result of lower conductivity (discussed below) due to reduced grain size and oxidation at nanocrystalline grain boundaries, which becomes less significant as the film thickness increases ${ }^{57}$. In situ SE measurements suggest that even the thinnest TiN films showed modest plasmonic behavior as grown (supplementary Figure S2); however, their plasmonic properties were significantly degraded upon exposure to air, presumably due to a reduction in free carrier density from film oxidation.

Interestingly, the thinnest films produced for this study still show a technologically useful level of conductivity $^{36}$, as shown by the VASE and van der Pauw measurements in Fig. 2c. This implies that even though the plasmonic behavior of these films is destroyed upon exposure to oxygen ${ }^{54,55,57}$, a number of conductive pathways exist at thicknesses close to the native oxide thickness ( 1-2 nm). Post-oxidation conductivity saturates for TiN films thicker than $\sim 10 \mathrm{~nm}$. This is a critically important factor when considering high surface area electrodes, which will require extremely thin films to maintain optical activity due to the inherent opacity of plasmonic TiN films. We are able to use these properties to fabricate high-porosity, transparent, conductive electrodes that are stable under ambient conditions. Furthermore, it is possible that a layer of $\operatorname{SiN}_{\mathrm{x}}$ could be used to passivate the TiN surface against oxidation and further enhance its ex situ properties. Finally, the van der Pauw measurements universally show slightly lower conductivity than the SE or VASE measurements. As a direct electrical measurement, the van der Pauw conductivity is strongly affected by grain boundary scattering, which will be significant in these nanocrystalline films. On the other hand, SE and VASE are all-optical measurements, and 


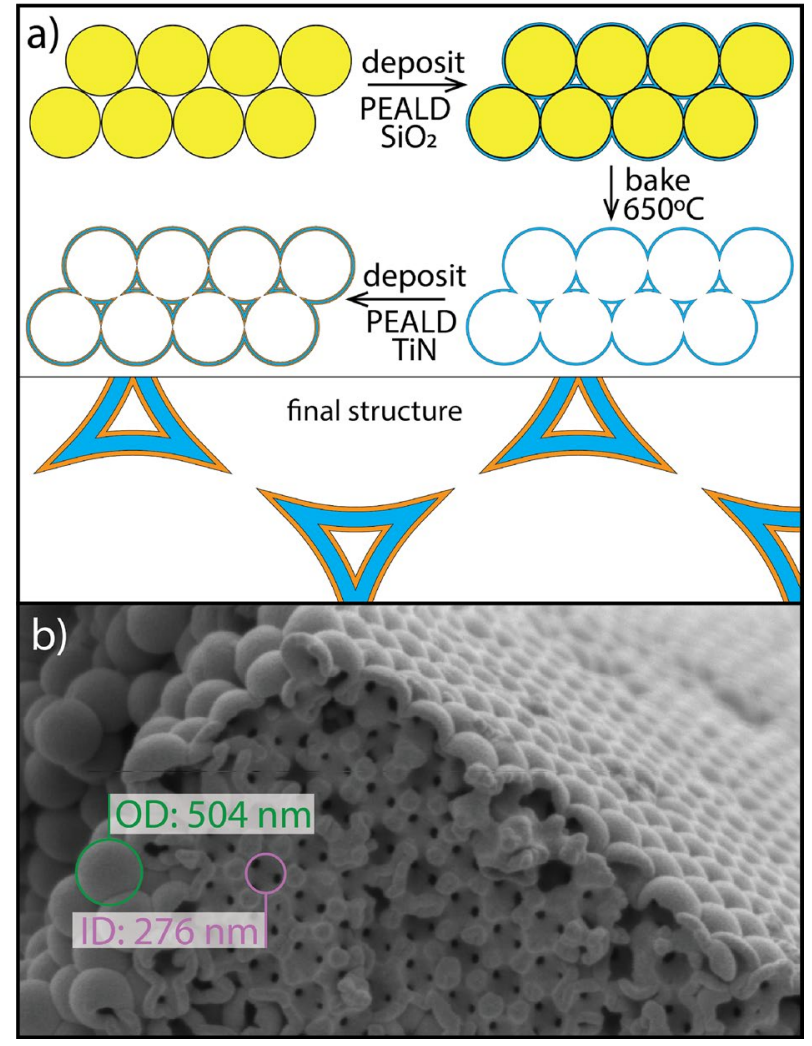

Figure 1. Inverse opal fabrication. Fabrication of TiN plasmonic, photonic, and conductive inverse opals. (a) Polystyrene beads in solution were evaporated onto a quartz slide and allowed to dry. These opals were partially infilled with a conformal layer of PEALD $\mathrm{SiO}_{2}$ and then the organic component was removed by annealing. The remaining $\mathrm{SiO}_{2}$ inverse opal was then partially infilled with a conformal layer of PEALD TiN. (b) A postanneal scanning electron micrograph of an inverse opal coated with $47 \mathrm{~nm}$ of TiN shows the complex, periodic, and high surface area conductive inverse opal lattice. Starting with a $390 \mathrm{~nm}$ polystyrene sphere, a $10 \mathrm{~nm} \mathrm{SiO}_{2}$ conformal coating and a $47 \mathrm{~nm}$ TiN conformal coating will yield an outer diameter of $504 \mathrm{~nm}$ (in green) and an inner diameter of $276 \mathrm{~nm}$ (in purple).

hence are relatively insensitive to grain boundary scattering of carriers. As such, this difference is more significant in thinner films with smaller grains.

With an understanding of the optical and electronic properties of the planar TiN thin films, we investigated the properties of the $\mathrm{SiO}_{2}$ and $\mathrm{SiO}_{2}$ /TiN inverse opals. Using variable-angle reflectometry (VAR), we were able to probe the angle-dependent optical properties of the bare $\mathrm{SiO}_{2}$ scaffold and the $3.5 \mathrm{~nm}$ TiN-coated structures, shown in Fig. 3a,b, respectively. In both structures, the dominant resonance shifts from approximately $2.5 \mathrm{eV}$ to $5 \mathrm{eV}$ as the measurement angle increases from $45^{\circ}$ to $80^{\circ}$, which is traced by gray lines. This shift is expected for opal or inverse opal structures, as the structure periodicity, which determines the optical response, varies as a function of the incident beam angle ${ }^{34,58}$. It is of particular note that the dominant photonic resonance is maintained in the TiN coated structure despite the presence of the conductive and plasmonic coating. The main modifications to the photonic response are a slight damping of the mode intensity and the appearance of two additional weak resonances at low energy (also traced by gray lines in Fig. 3b), likely due to new optical modes originating from the TiN coating. Examining individual spectra reveals the extent of modification induced by the TiN film. Figure $3 c$ shows the reflected intensity of the $\mathrm{SiO}_{2}$ and $\mathrm{SiO}_{2} / \mathrm{TiN}$ structures at $45^{\circ}$, where a small shift in the main resonance $(\sim 50 \mathrm{meV})$ has taken place, but the spectrum is largely unchanged. Figure $3 \mathrm{~d}$ shows that the resonance shift $(\sim 140 \mathrm{meV})$ becomes more significant at $65^{\circ}$, and resonance damping is clear, with $\sim 50 \%$ reduction in intensity. Increased effects from the presence of the TiN thin film are expected at glancing angles as the reflected light path length within the material increases, and therefore the number of $\mathrm{SiO}_{2} / \mathrm{TiN} /$ air interfaces, increases. At these angles, where the light passes through the most material, the photonic response is relatively unperturbed by the presence of the conductive TiN film. This property implies a general usefulness to this technique that will allow for the conversion of any porous photonic structure into a conductive electrode, with minimal impact on optical properties.

In order to understand the photonic-plasmonic interactions within the $\mathrm{SiO}_{2} / \mathrm{TiN}$ inverse opal structure, we compare the plasmonic resonance of the planar TiN films (which are not photonic and therefore decoupled) to the $\mathrm{SiO}_{2} / \mathrm{TiN}$ inverse opal photonic resonance. The plasmonic figure of merit (FOM) expresses the energydependent quality factor of the plasmonic resonance and is defined as the ratio of the real and imaginary part of the dielectric function $\left(\mathrm{FOM}=-\epsilon_{m}^{\prime} / \epsilon_{m}^{\prime \prime}\right)$. The plasmonic transition energy $\left(\mathrm{E}_{\mathrm{pl}}\right.$, where $\epsilon_{m}^{\prime}=0$ and becomes 


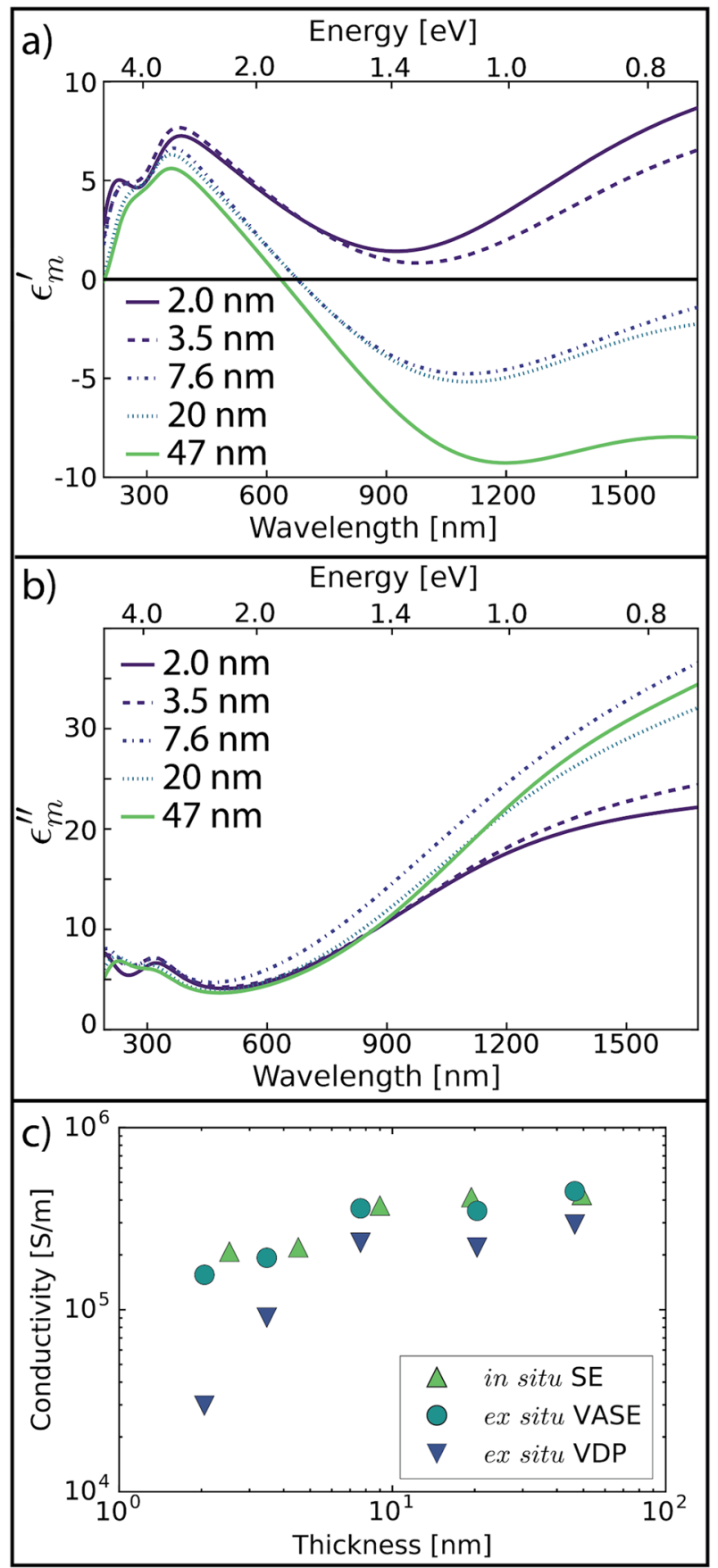

Figure 2. Dielectric function and conductivity. Optical and electronic properties of TiN thin films deposited on $\mathrm{SiO}_{2}$-coated silicon substrates. The real (a) and imaginary (b) parts of the dielectric function fit to ex situ VASE data for each TiN film thickness $(2-47 \mathrm{~nm})$. (c) Spectroscopic ellipsometry was also used to measure the film thickness and conductivity both in situ (green upright triangle) and after deposition and exposure to air using the VASE method (blue circle). These optical measurements of conductivity are compared to electrical transport measurements performed using the van der Pauw (VDP) method (inverted purple triangle).

non-negative) is the high-energy cutoff of the plasmonic response. As $\mathrm{E}_{\mathrm{pl}}$ moves towards and overlaps with the photonic resonance, there is the potential for maximizing both optical effects.

In Fig. $4 \mathrm{a}$, we show the FOM of a TiN sample $(\sim 7.6 \mathrm{~nm}$ thick) before and after thermal annealing at different temperatures. These measurements were taken on planar substrates that were deposited and annealed concurrently with the photonic structures. The non-annealed sample has an $\mathrm{E}_{\mathrm{pl}}$ of $1.75 \mathrm{eV}$, placing it far from the photonic resonance position $\left(\mathrm{E}_{\max }\right)$ of this structure at $2.45 \mathrm{eV}$, which is shown in Fig. $4 \mathrm{~b}$. By annealing the TiN films under a reducing atmosphere, we were able to modify and improve the plasmonic response of even extremely thin TiN films. In this case, annealing enhanced the FOM and blue-shifted $\mathrm{E}_{\mathrm{pl}}$ closer to the photonic 


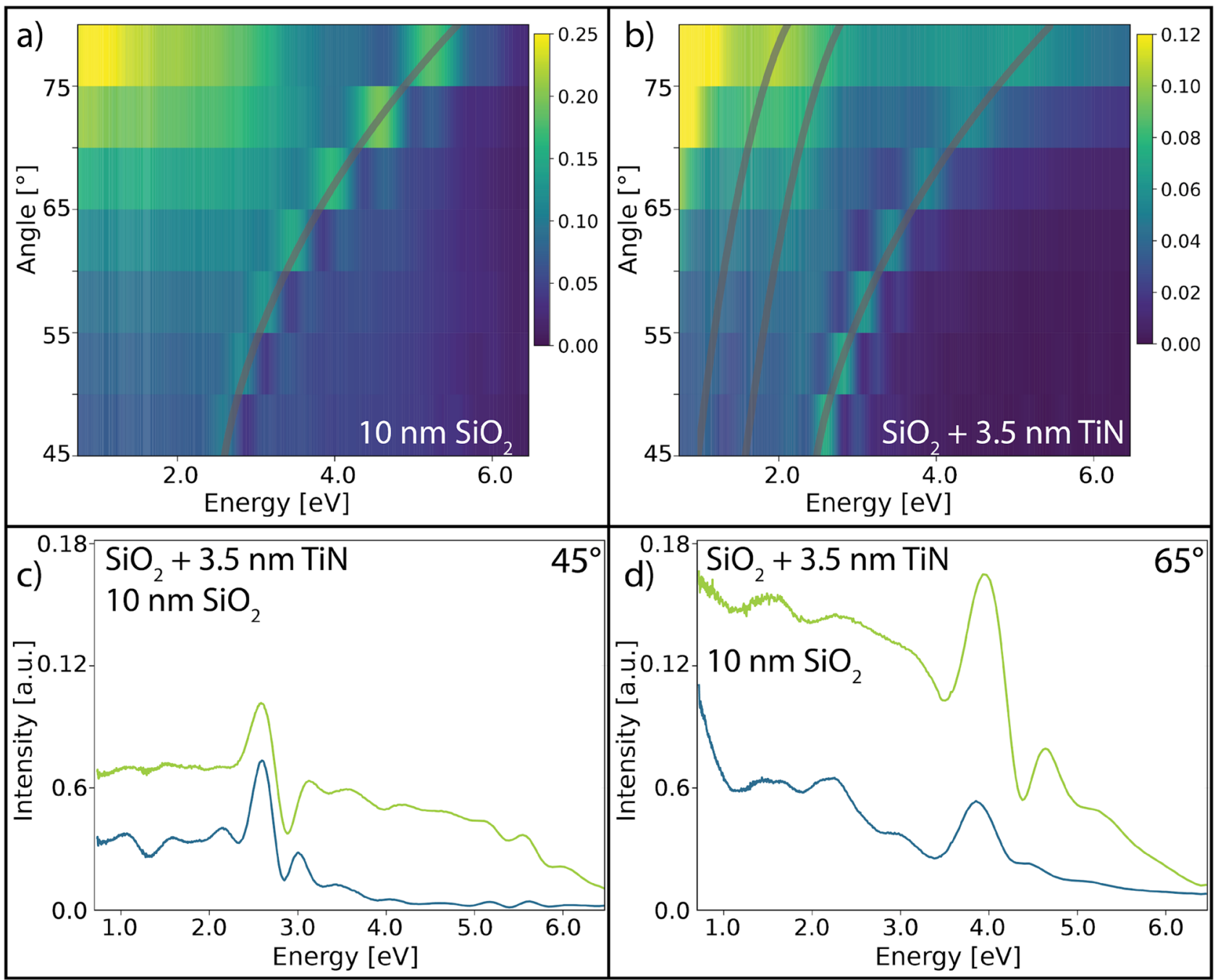

Figure 3. Inverse opal reflected intensity. Reflected intensity measured by VASE. (a) A strong photonic mode is present in the $10 \mathrm{~nm} \mathrm{SiO}$ inverse opal, which (b) is somewhat attenuated after coating with $3.5 \mathrm{~nm}$ of conductive and refractory TiN. Two additional weak low energy modes can be seen in (b) after TiN coating. Comparison of the reflected intensity of two inverse opal samples measured at (c) $45^{\circ}$ and (d) $65^{\circ}$. A small redshift in photonic resonance after TiN deposition is visible at $65^{\circ}$.

resonance. One would expect an increased plasmonic FOM to result in damping of the photonic resonance in the $\mathrm{SiO}_{2} / \mathrm{TiN}$ structure due increased reflectivity. However, upon annealing at $1100^{\circ} \mathrm{C}$, we see a large increase in the plasmonic FOM with minimal damping of the photonic resonance. Additionally, we observe a shift of $\mathrm{E}_{\mathrm{pl}}$ to $2.70 \mathrm{eV}(\Delta 0.95 \mathrm{eV})$ in the planar TiN films (Fig. 4a), but a less significant change to $\mathrm{E}_{\text {max }}$, now at $2.75 \mathrm{eV}$ $(\Delta 0.3 \mathrm{eV})$, in the $\mathrm{SiO}_{2} / \mathrm{TiN}$ structure (Fig. 4b). In Fig. 4c, we directly compare the effect of annealing on $\mathrm{E}_{\mathrm{pl}}$ and plasmonic resonance of the planar TiN films to the photonic resonance of the $\mathrm{SiO}_{2} / \mathrm{TiN}$ inverse opal structure. Both curves show a clear blue-shift upon annealing; however, the effect is more significant for $\mathrm{E}_{\mathrm{pl}}$ than $\mathrm{E}_{\max }$, likely due to crystallinity and purity improvement in the TiN from annealing. The shift in $\mathrm{E}_{\max }$ is likely due to densification of both the $\mathrm{SiO}_{2}$ and TiN, which induces small structural and dielectric response changes. Despite the likely structural changes taking place under these conditions, the thin film inverse opal with wall thicknesses of $25 \mathrm{~nm}$ $\left(\mathrm{TiN} / \mathrm{SiO}_{2} / \mathrm{TiN}-7.6 / 10 / 7.6 \mathrm{~nm}\right)$ and a height of approximately 4 microns ( 10 layers of $390 \mathrm{~nm}$ beads) remains stable up to $1100^{\circ} \mathrm{C}$. This allowed us to tune the plasmonic and photonic resonances into an overlapping regime with little, if any, degradation of optical properties of the photonic structure. This is a significant result because it implies that it is possible to decouple the plasmonic and photonic interactions within the $\mathrm{SiO}_{2} / \mathrm{TiN}$ inverse opal structure, enabling a highly porous, transparent electrode structure where the light trapped by the photonic structure can be paired with the plasmonic resonance energy of the TiN coating without damping effects.

In summary, we have demonstrated the ability to fabricate three-dimensional, plasmonically active photonic crystals using PEALD of TiN in an inverse opal-like structure. The TiN plasmonic resonance can be tuned via thermal treatment to be near the photonic resonance of the entire structure, allowing for overlapping optical modes to be present, which can allow one to take advantage of both photonic light guiding and plasmonic resonance enhancement without sacrificing the optical properties of either. We used planar TiN films, VASE, 

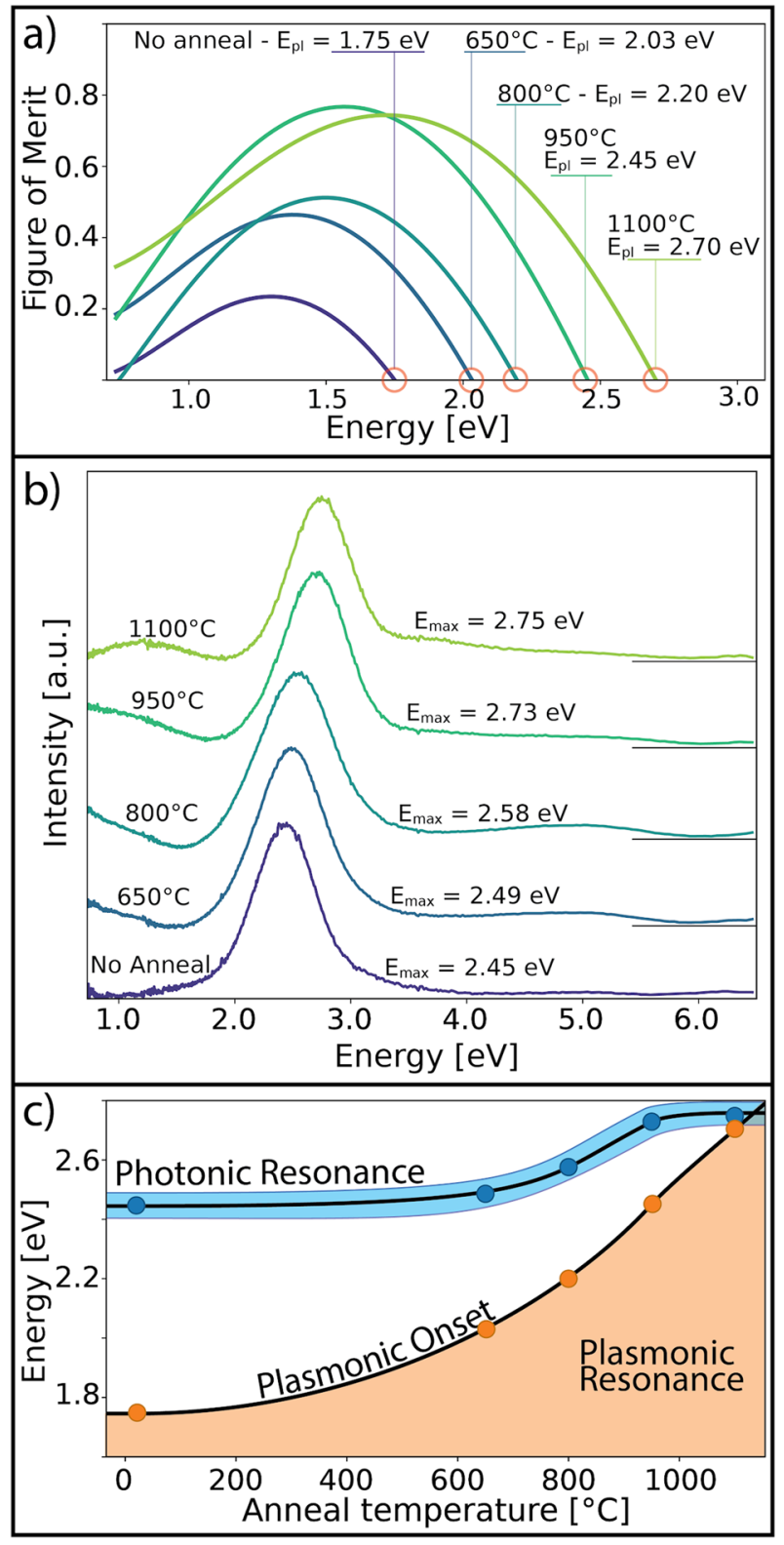

Figure 4. Annealing effects on planar TiN films and inverse opal samples. Annealing changes the plasmonic properties of the TiN. (a) The figures of merit (ratio of the real and imaginary parts of the dielectric function where the real part is negative) after each annealing temperature on a 150-cycle two-dimensional planar film demonstrate the evolution in film plasmonic quality in the visible and near infrared. The plasmonic transition energies $\left(\mathrm{E}_{\mathrm{pl}}\right)$ of the films blue-shift with increasing annealing temperature. (b) The $\mathrm{SiO}_{2} / \mathrm{TiN}$ photonic crystal resonance peak also blue-shifts with increasing annealing temperature, though to a lesser degree. (c) Overlapping the two trends-photonic and plasmonic resonance-show that with the right material properties, plasmonically and photonically active electrodes can be designed.

and van der Pauw measurements to provide insight into the electronic and plasmonic properties of the TiNcoated photonic crystal structures. Using different sized polystyrene beads as a template ${ }^{34}$ and other ALD film thicknesses to form the inverse opal could result in additional tuning of the plasmonic and photonic resonance overlap. The methods demonstrated in this work could be extended to more complex template structures, including three-dimensional chiral helices, in an industrially compatible manner using holographic lithography ${ }^{59-63}$. We believe such structures discussed herein could double as high surface area photoelectrodes for additional electronic functionality and find use in renewable energy systems and many other applications. 


\section{Materials and methods}

Inverse opals were grown through a multi-step assembly and coating process. First, self-assembled polystyrene (PS) opal structures were formed by evaporating colloidal solutions of PS beads onto quartz substrates using previously reported methods ${ }^{33}$ and as described below.

Polystyrene opal synthesis. Substrates (quartz, $1 \mathrm{~mm}$ thick, purchased from VWR) were cleaved into $1 \times 2 \mathrm{~cm}^{2}$ pieces and were cleaned in a three-step process: first, a 15-min sonication in an Alconox/deionized (DI) water solution, second, a 15-min sonication in pure DI water, and third, a 15-min sonication in a 1:1:1 acetone:ethanol:DI water solution. This cleaning process was followed by a 5 -min plasma treatment in air. Substrates were inspected between each step, and dust particles were removed with a flow of $\mathrm{N}_{2}$.

The slow-evaporation deposition of the PS bead opal templates is described in detail in our publication ${ }^{33}$ and also in an author's dissertation ${ }^{64}$. To summarize, $390 \mathrm{~nm}$ PS bead solutions (non-functionalized, $25 \mathrm{mg} / \mathrm{mL}$ in DI water, Bangs Labs) were diluted to $0.1 \mathrm{v} / \mathrm{v} \%$ using Millipore $\mathrm{R}$ water $\left(18.2 \mathrm{M} \Omega \mathrm{cm}, 25^{\circ} \mathrm{C}\right)$. Then, a few drops of a $1 \%$ solution of TritonX were added ( 3 drops $/ 8 \mathrm{~mL}$ ). The prepared substrates were each placed in a small vial ( $1.5 \mathrm{~cm}$ diameter and $4.5 \mathrm{~cm}$ height) tilted at a $60^{\circ}$ angle (where $0^{\circ}$ is horizontal). 16 vials were arranged in a 4 $\times 4$ grid on a hotplate. A 15-min sonication of the prepared PS bead solution was performed just before deposition onto the substrate. Then, $1.5 \mathrm{~mL}$ of the prepared PS bead solution were carefully dispensed into each vial. A crystallization dish ( $15.0 \mathrm{~cm}$ diameter and $7.5 \mathrm{~cm}$ height) was added to cover the hotplate, and filter paper was included in the bottom of the dish (now above the vials) to absorb and disperse condensation rather than allowing it to drip into the vials. The hotplate was then set to $50{ }^{\circ} \mathrm{C}$, and the humidity and evaporation rate were reproduced by always keeping a consistent amount of water (equal number of filled vials) in the enclosed deposition system. Once the deposition was complete, a 1-hr immersion in ethanol was performed to improve the crystallinity of the periodic aggregate samples. The samples were then carefully dried from the backside using a gentle flow of $\mathrm{N}_{2}$. To finish the samples, a 10 -min anneal was performed on a hotplate at $90^{\circ} \mathrm{C}$.

Inverse opal synthesis. Once the PS opals were fully dry, they were conformally coated with $10 \mathrm{~nm}^{\circ} \mathrm{SiO}_{2}$ using PEALD at $40^{\circ} \mathrm{C}$ using an Oxford Instruments FlexAL PEALD system (tris(dimethylamino)silane precursor, Sigma Aldrich). To remove the PS scaffold the samples were annealed at $650^{\circ} \mathrm{C}$ for $30 \mathrm{~min}$ in air inside a glass tube furnace (ThermoFisher Scientific Lindberg Blue M, 1" tube) to vaporize the PS, leaving behind the skeletal $\mathrm{SiO}_{2}$ structure, which maintained the original morphology. TiN was then deposited conformally onto the scaffold structure via PEALD at $300{ }^{\circ} \mathrm{C}$ in thicknesses ranging from $\sim 2 \mathrm{~nm}$ to $50 \mathrm{~nm}$ (tetrakis(dimethylamido)titanium precursor, Sigma Aldrich and a two-stage $\mathrm{N}_{2}$ then $\mathrm{H}_{2}$ plasma) using another previously reported recipe $\mathrm{e}^{56}$, resulting in highly porous photonic structures which maintain the original PS structure, as shown in Fig. $1 \mathrm{~b}$. TiN growth was monitored via in situ spectroscopic ellipsometry (J. A. Woollam Inc. M2000, 190-1690 nm or 0.73 to $6.9 \mathrm{eV}$.) on planar reference samples. Ex situ optical properties of the planar TiN films and inverse opals were measured via VASE and VAR (J. A. Woollam Inc. VASE M2000, 190-1690 nm or 0.73 to $6.9 \mathrm{eV}$.) and at angles ranging from $45^{\circ}$ to $75^{\circ}$ and $45^{\circ}$ to $80^{\circ}$, respectively, in $5^{\circ}$ increments. Measurements were analyzed using the CompleteEASE software package (J. A. Woollam Inc.). Conductivity of the planar TiN films was directly measured by the van der Pauw method (Ecopia HMS-5000) at room temperature.

Annealing of inverse opals and TiN films. This process is also described in an author's dissertation ${ }^{64}$. A quartz tube furnace (ThermoFisher Scientific Lindberg Blue M, 1" tube, $1100^{\circ} \mathrm{C}$ max) was used to perform anneals. Isopropyl alcohol was used to clean the quartz tube at its ends, the sealing attachments, and the quartz slide sample holder. Once the system was pumped to $1.5 \times 10^{-1}$ Torr, a leak check was performed by isolating the pump from the tube and confirming the tube's connections to the upstream gas flow did not have significant leaks. The system was pump-purged (to $1.5 \times 10^{-1}$ Torr, then filled with Ar) twice. $250 \mathrm{sccm} \mathrm{Ar}$ and $20 \mathrm{sccm} \mathrm{H}_{2}$ were flowed through the Ar-filled tube. Over 10's of minutes, the furnace temperature was ramped from room temperature $\left(\sim 20^{\circ} \mathrm{C}\right)$ to the annealing temperature as fast as possible, followed by a 1-hr anneal, and then cooled. When the samples were cool enough to remove from the furnace $\left(\leq 80-100{ }^{\circ} \mathrm{C}\right)$, the $\mathrm{H}_{2}$ gas flow was stopped, and the Ar was allowed to purge the system. Varying TiN coating thicknesses were annealed simultaneously under common conditions and always consistently ordered along the direction of gas flow through the tube with the thinnest samples (planar films on $\mathrm{SiO}_{2} / \mathrm{Si}$ ) upstream from the thickest samples (inverse opals on quartz) in order to prevent $\mathrm{H}_{2}$ flow shadowing over downstream samples.

Further details of both sample preparation and optical characterization are provided in the supplementary information.

Received: 27 November 2020; Accepted: 8 March 2021

Published online: 07 April 2021

\section{References}

1. Brongersma, M. L., Hartman, J. W. \& Atwater, H. H. Plasmonics: Electromagnetic energy transfer and switching in nanoparticle chain-arrays below the diffraction limit. MRS Proc. 582, 2 (1999)

2. Brongersma, M. L. Introductory lecture: Nanoplasmonics. Faraday Discuss. 178, 9-36 (2015).

3. Atwater, H. A. The promise of plasmonics. Sci. Am. 17, 56-63 (2007).

4. Kinsey, N. et al. Experimental demonstration of titanium nitride plasmonic interconnects. Opt. Express 22, 12238-12247 (2014).

5. Jeanmarie, D. L. Surface Raman Spectroelectrochemistry: Part I. Heterocyclic, aromatic, and aliphatic amines adsorbed on the anodized silver electrode. J. Electronanal. Chem. 84, 1-20 (1977).

6. Anker, J. N. et al. Biosensing with plasmonicnanosensors. Nat. Mater. 7, 442-453 (2008). 
7. Lindquist, N. C., Nagpal, P., McPeak, K. M., Norris, D. J. \& Oh, S.-H. Engineering metallic nanostructures for plasmonics and nanophotonics. Rep. Prog. Phys. 75, 036501 (2012).

8. Akimov, A. V. et al. Generation of single optical plasmons in metallic nanowires coupled to quantum dots. Nature 450, 402-406 (2007).

9. Oulton, R. F. et al. Plasmon lasers at deep subwavelength scale. Nature 461, 629-632 (2009).

10. Oulton, R. F., Sorger, V. J., Genov, D. A., Pile, D. F. P. \& Zhang, X. A hybrid plasmonic waveguide for subwavelength confinement and long-range propagation. Nat. Photonics 2, 496-500 (2008).

11. Atwater, H. A. \& Polman, A. Plasmonics for improved photovoltaic devices. Nat. Mater. 9, 205-213 (2010).

12. Khalifa, A. E. \& Swillam, M. A. Cheap and efficient plasmonic solar cell. Phys. Simul. Photonic Eng. Photovolt. Dev. https://doi.org/ $10.1117 / 12.2040776(2014)$.

13. Creel, E. B. et al. Directing selectivity of electrochemical carbon dioxide reduction using plasmonics. ACS Energy Lett. 4, 1098-1105 (2019).

14. Kim, Y. et al. Surface-plasmon-assisted photoelectrochemical reduction of $\mathrm{CO}_{2}$ and $\mathrm{NO}_{3}-$ on nanostructured silver electrodes. Adv. Energy Mater. 8, 1800363 (2018).

15. DuChene, J. S. et al. Optical excitation of a nanoparticle $\mathrm{Cu} / \mathrm{p}-\mathrm{NiO}$ photocathode improves reaction selectivity for $\mathrm{CO}_{2}$ reduction in aqueous electrolytes. Nano Lett. 20, 2348-2358 (2020).

16. Kumar, D., Lee, A., Lee, T., Lim, M. \& Lim, D.-K. Ultrafast and efficient transport of hot plasmonic electrons by graphene for Pt free, highly efficient visible-light responsive photocatalyst. Nano Lett. 16, 1760-1767 (2016).

17. Zhang, X., Liu, Y., Lee, S.-T., Yang, S. \& Kang, Z. Coupling surface plasmon resonance of gold nanoparticles with slow-photon-effect of $\mathrm{TiO}_{2}$ photonic crystals for synergistically enhanced photoelectrochemical water splitting. Energy Environ. Sci. 7, 1409 (2014).

18. Naldoni, A. et al. Broadband hot-electron collection for solar water splitting with plasmonic titanium nitride. Adv. Opt. Mater. 5, 1601031 (2017).

19. Mubeen, S. et al. An autonomous photosynthetic device in which all charge carriers derive from surface plasmons. Nat. Nanotechnol. 8, 247-251 (2013).

20. Linic, S., Christopher, P. \& Ingram, D. B. Plasmonic-metal nanostructures for efficient conversion of solar to chemical energy. Nat. Mater. 10, 911-921 (2011).

21. Challener, W. A. et al. Heat-assisted magnetic recording by a near-field transducer with efficient optical energy transfer. Nat. Photonics 3, 220-224 (2009).

22. Stipe, B. C. et al. Magnetic recording at $1.5 \mathrm{~Pb} \mathrm{~m}-2$ using an integrated plasmonic antenna. Nat. Photonics 4, 484-488 (2010)

23. Soukoulis, C. M. \& Wegener, M. Past achievements and future challenges in the development of three-dimensional photonic metamaterials. Nat. Photonics https://doi.org/10.1038/nphoton.2011.154 (2011).

24. López-García, M. et al. Enhancement and directionality of spontaneous emission in hybrid self-assembled photonic-plasmonic crystals. Small 6, 1757-1761 (2010).

25. Yablonovitch, E. Inhibited spontaneous emission in solid-state physics and electronics. Phys. Rev. Lett. 58, 2059-2062 (1987).

26. John, S. Strong localization of photons in certain disordered dielectric superlattices. Phys. Rev. Lett. 58, 2486-2489 (1987).

27. Ben Bakir, B. et al. Surface-emitting microlaser combining two-dimensional photonic crystal membrane and vertical Bragg mirror. Appl. Phys. Lett. 88, 081113 (2006).

28. Wierer, J. J. Jr., David, A. \& Megens, M. M. III-nitride photonic-crystal light-emitting diodes with high extraction efficiency. Nat. Photonics 3, 163 (2009).

29. Mihi, A. \& Míguez, H. Origin of light-harvesting enhancement in colloidal-photonic-crystal-based dye-sensitized solar cells. J. Phys. Chem. B 109, 15968-15976 (2005).

30. Deubel, M. et al. Direct laser writing of three-dimensional photonic-crystal templates for telecommunications. Nat. Mater. 3 , 444-447 (2004).

31. Saitoh, K. \& Koshiba, M. Highly nonlinear dispersion-flattened photonic crystal fibers for supercontinuum generation in a telecommunication window. Opt. Express 12, 2027 (2004).

32. Blanco, A. et al. Large-scale synthesis of a silicon photonic crystal with a complete three-dimensional bandgap near 1.5 micrometres. Nature 405, 437-440 (2000).

33. Gaulding, E. A. et al. Fabrication and optical characterization of polystyrene opal templates for the synthesis of scalable, nanoporous (photo)electrocatalytic materials by electrodeposition. J. Mater. Chem. A Mater. Energy Sustain. 5, 11601-11614 (2017).

34. Aguirre, C. I., Reguera, E. \& Stein, A. Tunable colors in opals and inverse opal photonic crystals. Adv. Funct. Mater. 20, 2565-2578 (2010).

35. Arsenault, A. C., Puzzo, D. P., Manners, I. \& Ozin, G. A. Photonic-crystal full-colour displays. Nat. Photonics 1, 468 (2007).

36. Imai, Y. et al. Electrically conductive polymeric photonic crystals. Soft Matter 8, 6280 (2012).

37. O'Sullivan, F., Celanovic, I. \& Jovanovic, N. Optical characteristics of one-dimensional $\mathrm{Si}_{/} / \mathrm{SiO}_{2}$ photonic crystals for thermophotovoltaic applications. J. Appl. 2, 3 (2005).

38. Wang, L. P. \& Zhang, Z. M. Wavelength-selective and diffuse emitter enhanced by magnetic polaritons for thermophotovoltaics. Appl. Phys. Lett. 100, 063902 (2012).

39. Nam, Y. et al. Solar thermophotovoltaic energy conversion systems with two-dimensional tantalum photonic crystal absorbers and emitters. Sol. Energy Mater. Sol. Cells 122, 287-296 (2014).

40. Zhang, L., Reisner, E. \& Baumberg, J. J. Al-doped ZnO inverse opal networks as efficient electron collectors in BiVO 4 photoanodes for solar water oxidation. Energy Environ. Sci. 7, 1402-1408 (2014).

41. Kim, J.-H. et al. Ni-NiO core-shell inverse opal electrodes for supercapacitors. Chem. Commun. 47, 5214 (2011).

42. Zang, X. et al. Titanium disulfide coated carbon nanotube hybrid electrodes enable high energy density symmetric pseudocapacitors. Adv. Mater. 30, 1704754 (2018).

43. Moroz, A. Three-dimensional complete photonic-band-gap structures in the visible. Phys. Rev. Lett. 83, 5274-5277 (1999).

44. Boltasseva, A. \& Shalaev, V. M. All that glitters need not be gold. Science 347, 1308-1310 (2015).

45. Jang, D., Meza, L. R., Greer, F. \& Greer, J. R. Fabrication and deformation of three-dimensional hollow ceramic nanostructures. Nat. Mater. 12, 893-898 (2013).

46. Kang, S.-B. \& Lee, S.-I. Method of and apparatus for forming a metal interconnection in the contact hole of a semiconductor device. Patent (2000).

47. Sneh, O. Radical-assisted sequential CVD. US Patent (2001).

48. Scharrer, M., Wu, X., Yamilov, A., Cao, H. \& Chang, R. P. H. Fabrication of inverted opal ZnO photonic crystals by atomic layer deposition. Appl. Phys. Lett. 86, 151113 (2005).

49. Lee, J.-H. et al. Layer-by-layer photonic crystal fabricated by low-temperature atomic layer deposition. Appl. Phys. Lett. 90,151101 (2007).

50. Chen, C. T. et al. Very high refractive index transition metal dichalcogenide photonic conformal coatings by conversion of ALD metal oxides. Sci. Rep. 9, 2768 (2019).

51. Üpping, J. et al. Transparent conductive oxide photonic crystals on textured substrates. Photonics Nanostruct. Fundam. Appl. 9, 31-34 (2011).

52. Rugge, A., Becker, J. S., Gordon, R. G. \& Tolbert, S. H. Tungsten nitride inverse opals by atomic layer deposition. Nano Lett. 3, 1293-1297 (2003). 
53. King, J. S. et al. High-filling-fraction inverted ZnS opals fabricated by atomic layer deposition. Appl. Phys. Lett. 83, 2566-2568 (2003).

54. Langereis, E. et al. In situ spectroscopic ellipsometry as a versatile tool for studying atomic layer deposition. J. Phys. D Appl. Phys. 42, 073001 (2009).

55. Langereis, E., Heil, S. B. S., van de Sanden, M. C. M. \& Kessels, W. M. M. In situ spectroscopic ellipsometry study on the growth of ultrathin TiN films by plasma-assisted atomic layer deposition. J. Appl. Phys. 100, 023534 (2006).

56. Otto, L. M. et al. Plasma-enhanced atomic layer deposition for plasmonicTiN. Nanophotonic Mater. XIII https://doi.org/10.1117/ 12.2238340 (2016).

57. Miikkulainen, V., Leskelä, M., Ritala, M. \& Puurunen, R. L. Crystallinity of inorganic films grown by atomic layer deposition: Overview and general trends. J. Appl. Phys. 113, 021301 (2013).

58. Schroden, R. C., Al-Daous, M., Blanford, C. F. \& Stein, A. Optical properties of inverse opal photonic crystals. Chem. Mater. 14, 3305-3315 (2002).

59. Arpin, K. A. et al. Multidimensional architectures for functional optical devices. Adv. Mater. 22, 1084-1101 (2010).

60. Lu, C. \& Lipson, R. H. Interference lithography: A powerful tool for fabricating periodic structures. Laser Photonics Rev. 4, 568-580 (2009).

61. Chen, Y. C., Geddes, J. B., Lee, J. T., Braun, P. V. \& Wiltzius, P. Holographically fabricated photonic crystals with large reflectance. Appl. Phys. Lett. 91, 241103 (2007).

62. Chen, Y.-C., Geddes, J. B. 3rd., Yin, L., Wiltzius, P. \& Braun, P. V. X-ray computed tomography of holographically fabricated threedimensional photonic crystals. Adv. Mater. 24, 2863-2868 (2012).

63. Geddes, J. B., III. System and method for industrial scale continuous holographic lithography. US Patent (2018).

64. Otto, L. M. Engineering Materials and Characterization Methods for Mass-Produced Plasmonic Devices (University of Minnesota, 2017).

\section{Acknowledgements}

Work at the Molecular Foundry was supported by the Office of Science, Office of Basic Energy Sciences, of the U.S. Department of Energy under contract no. DE-AC02-05CH11231. E.A.G. and F.M.T. acknowledge support from the Joint Center for Artificial Photosynthesis, a DOE Energy Innovation Hub, supported through the Office of Science of the U.S. Department of Energy under award number DE-SC0004993. L.M.O. acknowledges support from the National Science Foundation Graduate Research Fellowship Program under Grant no. 00039202.

\section{Author contributions}

A.M.S., S.A., F.M.T., A.T.H, and B.J.H.S. supervised the work. A.M.S., C.T.C., S.A., A.T.H. and L.M.O. designed the PEALD processes. L.M.O. performed the PEALD on the PS opals at the Molecular Foundry, and the PS opals were synthesized by E.A.G. at the Joint Center for Artificial Photosynthesis. L.M.O. performed the van der Pauw measurements and thermal treatments at the Molecular Foundry with assistance from C.T.C. and T.R.K. L.M.O. performed the VASE measurements at the Joint Center for Artificial Photosynthesis with assistance from E.A.G. L.M.O. and D.F.O. collected the SEM images. L.M.O., A.M.S., and S.A. performed the data analysis. L.M.O. and A.M.S. wrote the manuscript with input from all authors.

\section{Competing interests}

The authors declare no competing interests.

\section{Additional information}

Supplementary Information The online version contains supplementary material available at https://doi.org/ 10.1038/s41598-021-86813-y.

Correspondence and requests for materials should be addressed to A.M.S.

Reprints and permissions information is available at www.nature.com/reprints.

Publisher's note Springer Nature remains neutral with regard to jurisdictional claims in published maps and institutional affiliations.

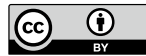

Open Access This article is licensed under a Creative Commons Attribution 4.0 International License, which permits use, sharing, adaptation, distribution and reproduction in any medium or format, as long as you give appropriate credit to the original author(s) and the source, provide a link to the Creative Commons licence, and indicate if changes were made. The images or other third party material in this article are included in the article's Creative Commons licence, unless indicated otherwise in a credit line to the material. If material is not included in the article's Creative Commons licence and your intended use is not permitted by statutory regulation or exceeds the permitted use, you will need to obtain permission directly from the copyright holder. To view a copy of this licence, visit http://creativecommons.org/licenses/by/4.0/.

This is a U.S. Government work and not under copyright protection in the US; foreign copyright protection may apply 2021 